NASZA DERMATOLOGIA Online OUR DERMATOLOGY Online

Source of Support: Nil Competing Interests: None

\section{DERMATOSCOPÍA: CONTRIBUCIÓN COMO MÉTODO PARA DEFINIR MÁRGENES QUIRÚRGICOS DE CARCINOMAS BASOCELULARES DE CARA, CUELLO Y TRONCO}

\section{DERMATOSCOPY: CONTRIBUTION AS A METHOD TO DEFINE SURGICAL MARGINS IN BASAL CELL CARCINOMAS OF THE FACE, NECK AND TRUNK}

\author{
Gabriela Martínez Braga, Rosalba Riveros, \\ Beatriz Di Martino Ortiz, Julio Recalde, Lourdes Bolla \\ Cátedra de Dermatología, Hospital de Clínicas, Facultad de Ciencias Médicas, \\ Universidad Nacional de Asunción, Paraguay
}

Corresponding author: Dr. Beatriz Di Martino Ortiz

beatrizdimartino@gmail.com

\begin{abstract}
Resumen
La escisión quirúrgica incompleta del Carcinoma Basocelular $(\mathrm{CBC})$ es posible por lo que métodos preoperatorios de marcación de márgenes tumorales es importante.

Guías para el manejo de los CBC han sido establecidas, sin embargo la determinación de una medida más adecuada de los márgenes es deseable, tal y como está establecido para los melanomas.

La Dermatoscopía es un procedimiento simple, barato, no invasivo que fue inicialmente utilizado para la evaluación de lesiones pigmentarias. Debido a que la misma técnica ha sido usada exitosamente en la evaluación de otras lesiones, como el CBC, hemos utilizado esta técnica para determinar los márgenes preoperatorios en este tipo de tumores.

El objetivo de este estudio fue comparar la determinación preoperatoria de los márgenes apropiados en la resección de los CBC usando dermatoscopía versus evaluación clínica, lo cual nos permitiría mejorar los resultados quirúrgicos.
\end{abstract}

\title{
Abstract
}

The incomplete excision of basal cell carcinoma (BCC) is possible that's why presurgical marking methods of tumor margins are important. Guides for the $\mathrm{CBC}$ handling have been established, however determining a better measure of the margins is desirable, as it is established for melanomas.

Dermoscopy is a simple, inexpensive, noninvasive method, which was initially used for the evaluation of pigmented lesions. Because the same technique has been used successfully in the evaluation of other injuries, as the CBC, we used this technique to determine preoperative margins in these tumors.

The aim of this study was to compare the preoperative determination of appropriate margins of BCC using dermatoscopy versus clinical assessment, which would allow us to improve surgical outcomes.

Palabras clave: Carcinoma Basocelular (CBC); márgenes quirúrgicos; dermatoscopía

Key words: basal cell carcinoma (BCC); surgical margins; dermatoscopy

\section{Cite this article:}

Gabriela Martínez Braga, Rosalba Riveros, Beatriz Di Martino Ortiz, Julio Recalde, Lourdes Bolla: Dermatoscopía: contribución como método para definir márgenes quirúrgicos de carcinomas basocelulares de cara, cuello y tronco. Our Dermatol Online. 2013; 4(1): 28-31

\section{Introduction}

El carcinoma basocelular $(\mathrm{CBC})$ es el tumor epitelial maligno más frecuente en la piel, correspondiendo al $75 \%$ de los cánceres cutáneos no melanoma [1,2]. Se presenta especialmente en la raza blanca, observándose un continuo incremento de la incidencia del mismo en las últimas décadas, involucrando a pacientes cada vez más jóvenes [3].
La tasa de curación depende del tamaño de la lesión, del sub-tipo histológico, la localización anatómica y la conducta terapéutica $[4,5]$.

Para elegir el tratamiento más apropiado, debe considerarse el tamaño del tumor, su ubicación, el tiempo de evolución del mismo y el tipo histológico que presenta. 
Otros factores importantes a considerar, son la edad del paciente, patologías concomitantes y la calidad de la piel peri tumoral, así como también, la experiencia del médico tratante $[1,6]$.

La extirpación quirúrgica del tumor es la terapia de primera elección, con altas tasas de efectividad, aunque en ocasiones es difícil determinar el límite real de la lesión debido a la extensión subclínica del tumor [7,8].

La dermatoscopía es una técnica no invasiva, de fácil aplicación, que inicialmente fue utilizada para lesiones pigmentarias de la piel y actualmente es utilizada para mejorar el diagnóstico clínico de CBC $[9,10]$.

\section{Objetivos}

- Evaluar la utilidad de la dermatoscopía en la obtención de márgenes libres de tumor durante la marcación pre quirúrgica de lesiones con diagnóstico de CBC.

- Comparar la determinación de márgenes quirúrgicos por dermatoscopía con la determinación de los mismos, hecha por observación puramente clínica.

\section{Material y Método}

Fueron incluidos pacientes con diagnóstico clínico presuntivo de $\mathrm{CBC}$ localizados en cara, cuello y tronco, que consultaron al servicio de Dermatología del Hospital de Clínicas, FCM-UNA, entre los meses de marzo de 2011 a enero de 2012, de ambos sexos, y con edades comprendidas entre 18 a 90 años, y que dieron su consentimiento escrito para participar del estudio.

Todas las lesiones fueron examinadas clínicamente y por dermatoscopía.

Los participantes fueron divididos en dos grupos:

- El grupo 1 consistía en aquellos pacientes con $\mathrm{CBC}$ extirpados quirúrgicamente y con diagnóstico confirmado por histopatología, cuyos márgenes fueron marcados pre quirúrgicamente por dermatoscopía, utilizando para ello un dermatoscopio portátil con polarización transversal fija (DERMLITE II MULTIESPECTRAL ${ }^{\mathrm{TM}}$ ), con lente de 10 aumentos y 32 LED.

- El grupo 2 incluía a pacientes con $\mathrm{CBC}$ extirpados quirúrgicamente y con diagnóstico confirmado por histopatología, cuyos márgenes fueron marcados pre quirúrgicamente mediante una evaluación clínica.

En cada caso se realizó la marcación con lápiz demográfico delimitando el tumor del tejido sano, considerando tejido sano a la piel sin patrones dermatoscópicos compatibles con CBC o piel con textura normal al evaluar clínicamente.

La incisión quirúrgica se realizó en el borde externo de cada marcación. El margen profundo se delimitó en todos los casos en el tejido celular subcutáneo. Todas las muestras fueron enviadas para estudio histopatológico.

Los especímenes fueron analizados histológicamente. Los mismos fueron fijados en formol neutro tamponado al $10 \%$ y procesados de manera rutinaria en procesador automático de tejidos, previa disección macroscópica siguiendo las recomendaciones del Colegio Americano de Patología (CAP). Las secciones fueron teñidas con hematoxilina y eosina.

Se realizó el informe de los márgenes, si se hallaban comprometidos o libres de lesión tumoral en regiones laterales y profundas.

Se realizó luego una comparación de los resultados histopatológicos de los márgenes entre ambos grupos utilizando la prueba estadística del chi cuadrado.

\section{Resultados}

El estudio incluyó 81 lesiones en 64 pacientes, 33 eran mujeres y 31 pacientes eran hombres, entre 18 a 90 años (promedio 58.7 años) todos con confirmación histopatológica de CBC.

El grupo 1: 49 lesiones en 37 pacientes: mujeres 20, hombres 17; con edades comprendidas entre 18 y 84 años (promedio 59.10 años). Los patrones histopatológicos de los CBC fueron: 43 nodulares, 5 multicéntricos superficiales, 1 infiltrante.

El grupo 2: 32 lesiones en 27 pacientes: mujeres 13, hombres 14; con edades comprendidas entre 30 y 90 años (promedio 58.2 años). Los patrones histopatológicos de los CBC fueron: 28 nodulares, 2 multicéntricos superficiales, 1 esclerodermiforme y 1 infiltrante.

Las lesiones estaban localizadas en las siguientes áreas anatómicas: nariz 17 , surco nasogeniano 7 , frente 3 , mejilla 16 , tronco 12 , región malar 14 , cervical 4 , región temporal 5 , región preauricular 2, tal y como se muestra en la Figura 1.

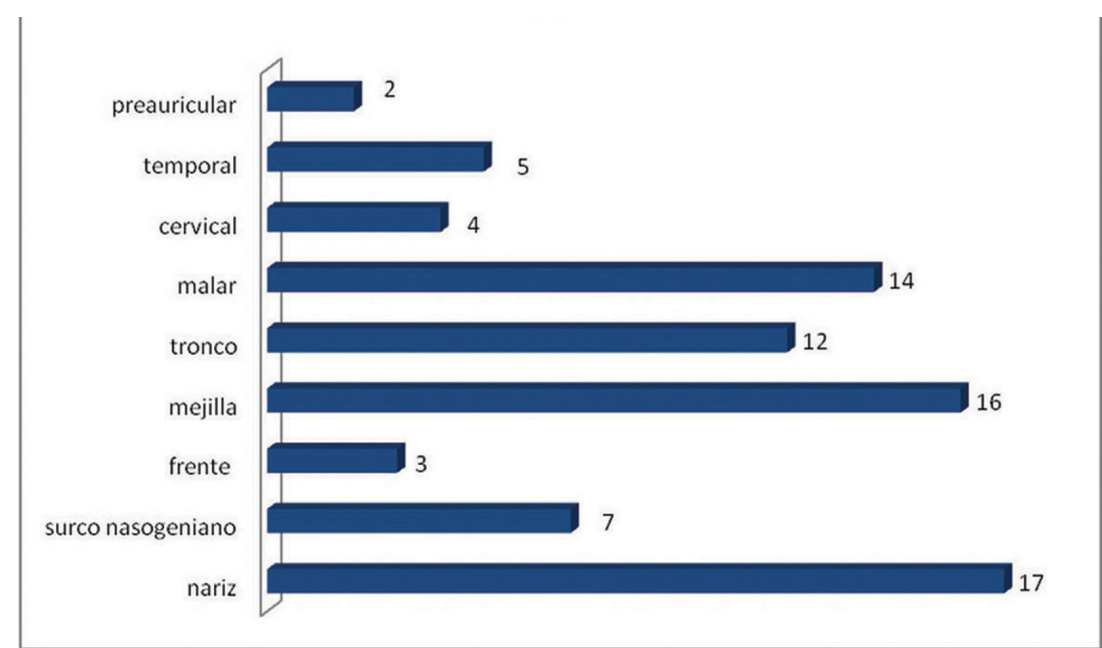

Figura I. Distribution according to location of the lesions. n: 81

Figure I. Distribución según localización de lesiones. n: 81 
De las 81 lesiones, 7 dieron resultados histopatológicos de márgenes comprometidos.

Los patrones histopatológicos encontrados se muestran en la Figura 2.

- En el grupo 1, de 49 lesiones extirpadas, 1 de ellas presentó márgenes laterales comprometidos, correspondiendo a un CBC multicéntrico superficial localizado en tronco.
- En el grupo 2, de 32 lesiones extirpadas, 6 de ellas presentaron márgenes comprometidos, siendo el patrón histopatológico nodular el predominante en 5 lesiones y el patrón esclerodermiforme en 1 lesión.

Comparando ambos grupos se encontró una diferencia estadísticamente significativa $(\mathrm{p}<0,01)$ en los resultados (Tabl. I).

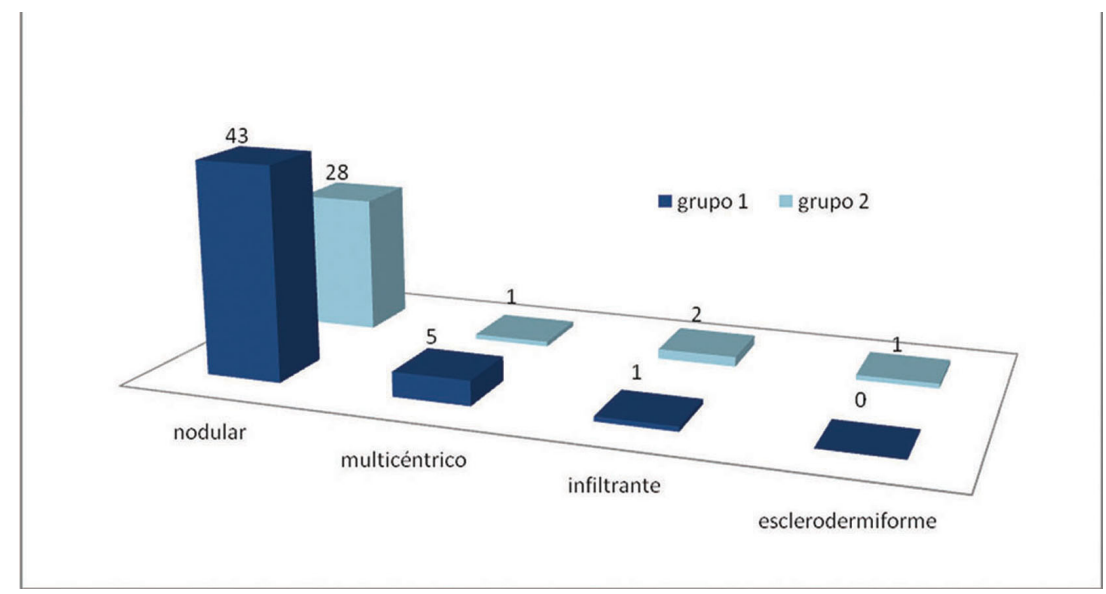

Figura II. Distribution according to histopathological pattern. $\mathrm{n}: 81$

Figure II. Distribución según patrón histopatológico. n: 81

\begin{tabular}{|l|cc|cc|}
\hline \multicolumn{1}{|c|}{ Patrón histopatológico } & \multicolumn{2}{c|}{$\begin{array}{c}\text { Grupo 1: } \\
\text { Evaluación por dermatoscopio }\end{array}$} & \multicolumn{2}{c|}{$\begin{array}{c}\text { Grupo 2: } \\
\text { Evaluación clinica }\end{array}$} \\
& Lesiones & Margen positivo & Lesiones & Margen positivo \\
\hline Nodular & 43 & 0 & 28 & 5 \\
\hline Multicéntrico superficial & 5 & 1 & 2 & 0 \\
\hline Esclerodermiforme & 43 & 0 & 1 & 1 \\
\hline Infiltrate & 43 & 0 & 1 & 0 \\
\hline
\end{tabular}

Tabla I. Comparación de los márgenes de resección quirúrgicos del CBC por dermatoscopía y clínica Table I. Comparison of surgical resection marginsof BCC by dermoscopy and clinical assessment

\section{Discusión}

El $\mathrm{CBC}$ es un tumor maligno de piel de crecimiento lento, localmente invasivo. Incompletamente escisionado, especialmente cuando está localizado en la cara, es más fácil su recurrencia. Por esta razón, la determinación adecuada de los márgenes de $\mathrm{CBC}$ es crucial en la evaluación preoperatoria del paciente $[9,11]$.

Para el tratamiento de los $\mathrm{CBC}$ existen varias alternativas terapéuticas como criocirugía, terapia fotodinámica y cirugía convencional, siendo esta última la más utilizada $[10,12,13]$. La importancia de obtener márgenes libres de tumor es evitar las recidivas. Se reportan tasas de 4 a $7 \%$ de márgenes comprometidos tras cirugía convencional en unidades de cirugía británicas y $6 \%$ en dos estudios de Australia $[10,12]$. Aunque con la cirugía de Mohs se observan tasas de recidivas menores, ésta no es utilizada en todos los centros, ya sea por costo o por no disponer de la infraestructura, como en el nuestro.

En estudios retrospectivos previos, CBC con escisión incompleta van de un rango de $4 \%$ a $16.6 \%$ con la cirugía tradicional. Debido a que en muchos centros dermatológicos usan la cirugía tradicional debido a ser más simple, barata y más corta con respecto a la cirugía de Mohs, el consenso principal sigue siendo como obtener márgenes pre quirúrgicos adecuados del tumor $[9,14,15]$.

Es necesario encontrar técnicas que permitan realizar marcaciones seguras de los márgenes. Se han realizado dos trabajos con excelentes resultados demostrando la utilidad de la dermatoscopía en la marcación de márgenes pre quirúrgicos de $\mathrm{CBC}[4,16,17]$.

En una serie estudiada sobre $\mathrm{CBC}$, se encontró que el $38 \%$ de las mujeres y el $25 \%$ de los hombres menores de 35 años, con diagnóstico de $\mathrm{CBC}$, tenían un tipo histológico de comportamiento agresivo, en oposición a los mayores de 60 años con tipos histológicos más circunscritos, concluyendo entonces, que los individuos más jóvenes, son propensos a desarrollar $\mathrm{CBC}$ con patrones de crecimiento más agresivos [18].

Diferentes métodos han sido propuestos, pero la dermatoscopía es, en nuestra opinión con los resultados obtenidos en nuestro estudio, el método más simple, más reproducible, seguro, barato y útil para obtener menores tasas de márgenes comprometidos $[19,20]$. 


\section{Conclusiones}

1. La dermatoscopia es un método de fácil aplicación que demostró ser más efectivo en relación a la observación clínica aislada en la asignación de los márgenes quirúrgicos en cirugías convencionales de $\mathrm{CBC}$.

2. Este hecho es relevante y estadísticamente significativo, y con la utilización de este método se evitarían numerosas recidivas tumorales, sobre todo en paciente jóvenes, donde muchos estudios demuestran que suelen tener tipos histológicos de comportamiento agresivo, en oposición a los mayores de 60 años con tipos histológicos más circunscritos.

\section{REFERENCIAS BIBLIOGRÁFICAS}

1. Negrin M: Carcinoma basocelular. Dermatol Venezol. 2008;46:416.

2. Weedon D: Tumores de la epidermis. En: Piel Patología. $1^{\circ}$ Ed. Madrid: Marban Libros, S.L. 2002. pp. 635-72.

3. Consenso sobre Carcinoma basocelular y carcinoma espinocelular. Guía de recomendaciones 2005. Consenso de la sociedad argentina de dermatología. Recuperado el 10 de agosto de 2008, en: http:// www.sad.org.ar/revista/pdf/basoespino.pdf

4. Eney M, Paschoal F, Valdés R: Contribución de la dermatoscopia en el establecmiento de márgenes quirúrgicos mínimos para la resección de carcinoma basocelular de cara y cuero cabelludo. Med Cutan Iber Lat Am 2011;39:205-28.

5. Robinson J, Fisher S: Recurrent basal cell carcinoma after incomplete resertion. Arch Dermatol. 2000;136:1318-24.

6. Odom R, James W, Berger T: Nevus, neoplasias y quistes epidérmicos. Andrew's Dermatología clínica. $9^{\circ} \mathrm{Ed}$. Philadelphia: Marban Libros. 2004. pp. 800-68.

7. Dandurand M, Petit T, Martel P, Guillot B: Management of basal cell carcinoma in adults. Clinical practice guidelines. Eur J Dermatol. 2006;16:394-401.
8. Sterry W. Guidelines: the management of basal cell carcinoma. Developed by the guideline subcommitte of the European dermatology forum. Eur J Dermatol. 2006;16:467-75.

9. Carducci M, Bozzetti M, Foscolo AM, Betti R: Margin detection using digital dermatoscopy improves the performance of traditional surgical excision of basal cell carcinomas of the head and neck. Dermatol Surg. 2011;37:280-5.

10. Menzies SW: Dermoscopy of pigmented basal cell carcinoma. Clin Dermatol. 2002;20:268-9.

11. Saldanha G, Fletcher, Slater DN: Basal cell carcinoma: a dermatopathological and biological update. $\mathrm{Br} \mathrm{J}$ Dermatol. 2003;148:195-202.

12. Charlin G, Serrano J, Del Pozo J: Carcinoma Basocelular. Guías Clínicas. 2005;5:305-8.

13. Kuijper D, Thiessen M, Nuemann M: Basal cell carcinoma: treatment options and prognosis, a scientific approach to the common malignancy. Am J Clin Dermatol. 2002;3:247-59.

14. Farhi D, Dupin N, Palangié A, Carlotti A, Avril MF: Incomplete excision of basal cell carcinoma: rate and associated factors among 362 consecutive cases. Dermatol Surg. 2007;33:1207-14.

15. Bogdanov-Berezovsky A, Cohen AD, Glesinger R, Cagnano E, Krieger Y, Rosenberg L: Risk factors for incomplete excision of basal cell carcinomas. Acta Derm Venereol. 2004;84:44-7.

16. Galimberti G, Montaño P: Cirugía micrográfica de Mohs en el tratamiento de carcinoma basocelular. Actas Dermosifiliogr. 2010;101:853-7.

17. Cabo H: Lesiones no melanocíticas. Carcinoma Basocelular. Dermatoscopia. Buenos Aires: Ed. Journal. 2008;7:83-91.

18. Telfer N: Guidelines: the management of basal cell carcinoma. British Journal of Dermatology. 2008;159:35-48.

19. Malvehy J, Llambrich À, Puig S: Signos guía en el diagnóstico diferencial en dermatoscopia. Piel. 2003;18:85-91.

20. Terushkin V, Wang SQ: Mohs surgery for basal cell carcinoma assisted by dermoscopy: report of two cases. Dermatol Surg. 2009;35:1-5. 